Gray, D. and Le Kernec, J. (2020) Scan Performance of Small Spherical RetroReflectors. In: 2019 International Radar Conference, Toulon, France, 23-27 Sept 2019, ISBN 9781728126609 (doi:10.1109/RADAR41533.2019.171391)

There may be differences between this version and the published version. You are advised to consult the publisher's version if you wish to cite from it.

\title{
http://eprints.gla.ac.uk/184545/
}

Deposited on: 18 April 2019

Enlighten - Research publications by members of the University of Glasgow http://eprints.gla.ac.uk 


\section{Scan performance of small spherical retro-reflectors}

D. Gray

Electrical Engingeering

XJTLU

Suzhou, China

derek.gray@xjtlu.edu.cn

\section{J. Le Kernec}

Electrical Engineering

Glasgow University

Glasgow, Scotland

julien.lekernec@glasgow.ac.uk

\begin{abstract}
A number of historical small spherical lens retroreflectors were simulated in a commercially available antenna simulator. This $1 \mathrm{dBm}^{2}$ class retro-reflectors had radii of 2 free space wavelengths, allowing the use of a minimal number of layers to approximate the theoretical Luneburg profile. It was found that further simplification to a 2-layer lens or a single custom material lens were practical. In both cases, RCS of $1.5 \mathrm{dBm}^{2}$ were achieved with $3 \mathrm{~dB}$ scan ranges of $130^{\circ}$.
\end{abstract}

Keywords—component, formatting, style, styling, insert

\section{INTRODUCTION}

Passive retro-reflectors reflect the majority of incident energy back in the direction of the illuminator, without the need for an external power supply [1]. These retro-reflectors are used as beacons to mark the edges of aircraft runways, to augment small vessels by making a larger return, and for calibrating radar measurements to an absolute value.

For student radar projects, having low cost in-house manufactured calibration targets would be advantageous. In the past, metal spheres were used to provide absolute radar cross section (RCS) data for calibration, but have a relatively low backscatter as most of the incident energy is reflected in directions other than that of the illuminator. In contrast, a Luneburg lens fitted with a reflective cap reflects all of the incident energy in phase so the RCS is the same as that of a flat plate. A good description of the use of Luneburg lens retro-reflectors in the field for calibrating soil moisture measurements during a lengthy measurement campaign was given in [2].

Theoretically, a Luneburg lens is a sphere that has relative permittivity $\varepsilon_{\mathrm{r}}=2$ at the centre and a continuous decrease with radial position to $\varepsilon_{\mathrm{r}}=1$ at the sphere surface. As such, the Luneburg lens is a limiting case having surface $\varepsilon_{\mathrm{r}}=1$; non-Luneburg lenses having surface $\varepsilon_{\mathrm{r}}>1$ to be built from natural plastics can be designed using either closed form equations or full wave software. In either case, realising the continuous $\varepsilon_{\mathrm{r}}$ profile in a sphere is impossible, so the lens $\varepsilon_{\mathrm{r}}$ profile is discretised into a series of unique $\varepsilon_{\mathrm{r}}$ steps and the lens built from thin concentric spherical shells, Figure 1. However, from prior work on lens antennas for communications systems it is understood that single material homogeneous spherical lenses give adequate performance up to about 5 wavelength $\left(\lambda_{0}\right)$ radius [3], obviating the need for the expensive concentric shell structure for Gain below $28 \mathrm{dBi}$. For the $28 \mathrm{dBi}$ to $35 \mathrm{dBi}$ Gain range, a 2-layer crosslinked polystyrene (xPS) core with polyethylene (PE) outer layer lens has proven successful [4]. The only disadvantage of this type is the relatively high mass compared to Luneburg lenses due to the specific gravity of the natural plastics. The aim for this work is to test homogeneous and the 2-layer $\mathrm{xPS}$-PE lenses as spherical retro-reflectors.

The angular arc of the metallic cap determines the angular range over which the lens gives a high value RCS. It is normal practise to use a $140^{\circ}$ metallic cap which gives a high RCS across about $120^{\circ}$, hence sets of 3 lenses been used to give full $360^{\circ}$ coverage in the horizontal plane for marine buoys and light craft. A $140^{\circ}$ metallic cap is illustrated in Figure 1, and was used in all simulations presented here.

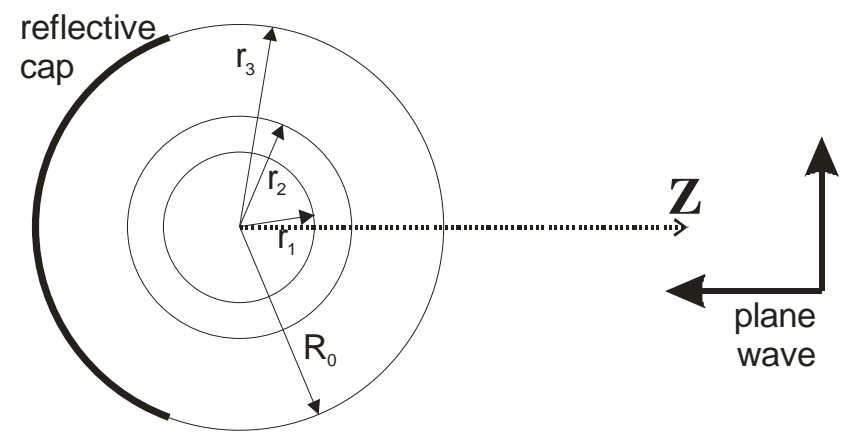

Fig. 1. Spherical lens topology simulated in FEKO ${ }^{\mathrm{TM}}$.

Here the commercially available Method of Moments antenna simulator $\mathrm{FEKO}^{\mathrm{TM}}$ using Higher Order Basis Function curvilinear elements was used to model the response to a linearly polarised plane wave of 2 historical 2 wavelength radius spherical retro-reflector lenses and some derivative designs. Material losses were not considered in the $\mathrm{FEKO}^{\mathrm{TM}}$ simulations. The centre frequency was $12 \mathrm{GHz}$ to be compatible with existing experimental equipment. The numerical simulation results were compared to the theoretical free space radar cross section of a metallic sphere was $-11.1 \mathrm{dBm}^{2}$ and for a spherical lens $0.9 \mathrm{dBm}^{2}$.

\section{OMNI-DiRECTIONAL REFLECTOR}

As an alternative to a Luneburg lens with a reflective cap, a lens having total internal reflection was designed, built and tested [5]. The advantage of this lens was that it would give a constant RCS independent of illumination incident angle, not having a limited angular range dictated by a spherical cap. In stark contrast to the spherical lens designs mentioned above, this theoretical lens design had infinite $\varepsilon_{\mathrm{r}}$ at the sphere centre, with the permittivity falling away rapidly with increasing lens radius to $\varepsilon_{\mathrm{r}}=1$ at the lens surface, Figure 2. The $\varepsilon_{\mathrm{r}}$ profile was discretised into constant $\varepsilon_{\mathrm{r}}$ steps so that the lens could be built from concentric spherical shells of unique materials, as described above. The original structure built and tested had a small high $\varepsilon_{\mathrm{r}}$ ceramic core [5], which was simplified in the second version to a metallic sphere, Figure 2. The latter version was simulated here.

As the effect of the number of layers upon lens RCS was not discussed in [5], it was investigated here. The lens profile outside of the $4.8 \mathrm{~mm}$ radius $\left(0.0952 \mathrm{R}_{0}\right)$ metallic core was discretised in 4, 6, 8 and 10 equal $\varepsilon_{\mathrm{r}}$ steps here, Figure 2. These 4 lenses were illuminated by a linearly polarised plane wave coming from $\theta=0^{\circ}$ in $\mathrm{FEKO}^{\mathrm{TM}}$ and the RCS in 
the principal and $\phi=45^{\circ}$ plane were noted. As the number of layers was increased, the $\theta=0^{\circ}$ RCS increased to an apparent asymptote equal to the RCS of an equal sized metallic sphere, Figure 3. This was an unexpectedly poor result.

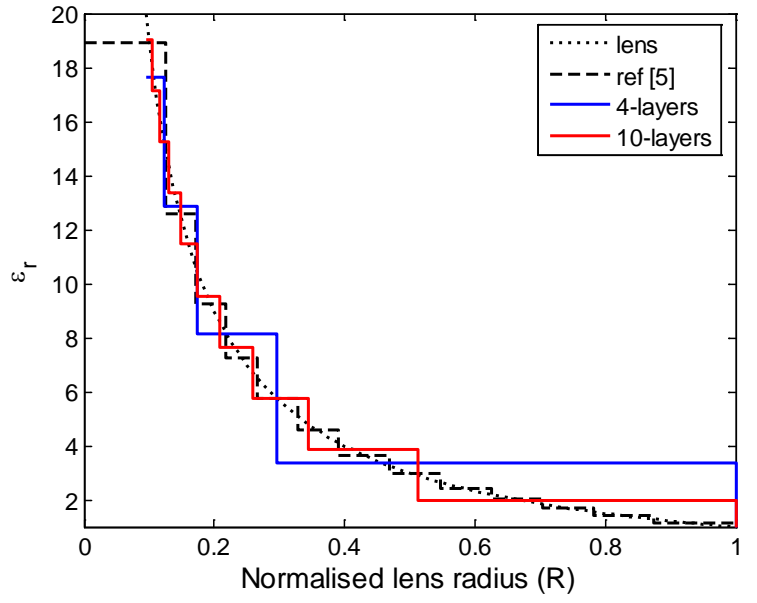

Fig. 2. Total internal reflection lens $\varepsilon_{\mathrm{r}}$ radial variation curve and discretised versions.

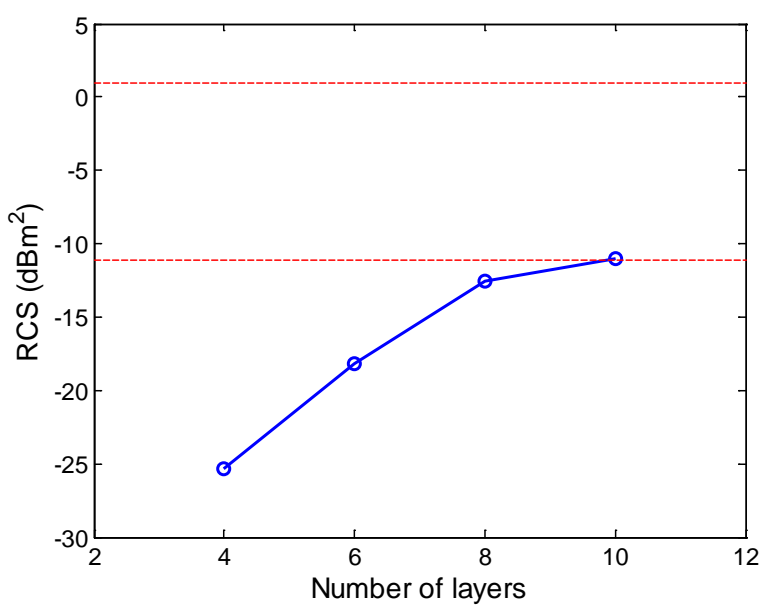

Fig. 3. Effect of number layers on total internal reflection lens $\theta=0^{\circ}$ RCS.

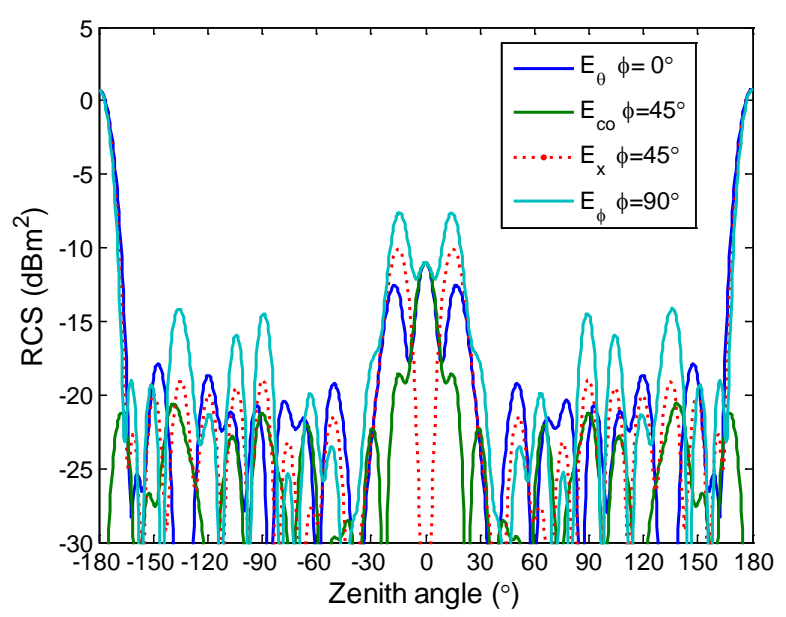

Fig. 4. Principal plane RCS patterns of 10-layer total internal reflection lens.
Examining the RCS patterns of the 10-layer implementation, it showed 3 major types of loss in the radiation pattern around the $\theta=0^{\circ}$ direction that led to the poor RCS result, Figure 4. In the E-plane $\left(\phi=0^{\circ}\right)$, the first side lobes were of almost equal amplitude as the mainlobe. In the $\phi=45^{\circ}$ plane, the cross-polarised radiation component had a higher amplitude than the co-polarised component. In the H-plane $\left(\phi=90^{\circ}\right)$, the mainlobe was split by a null in the $\theta=0^{\circ}$ direction. Overall, this lens type, as a circular aperture, had poor illumination and lost a significant amount of energy into the cross-polarised component in the $\phi=45^{\circ}$ plane. A metallic sphere would be a lower cost option for an equal RCS response.

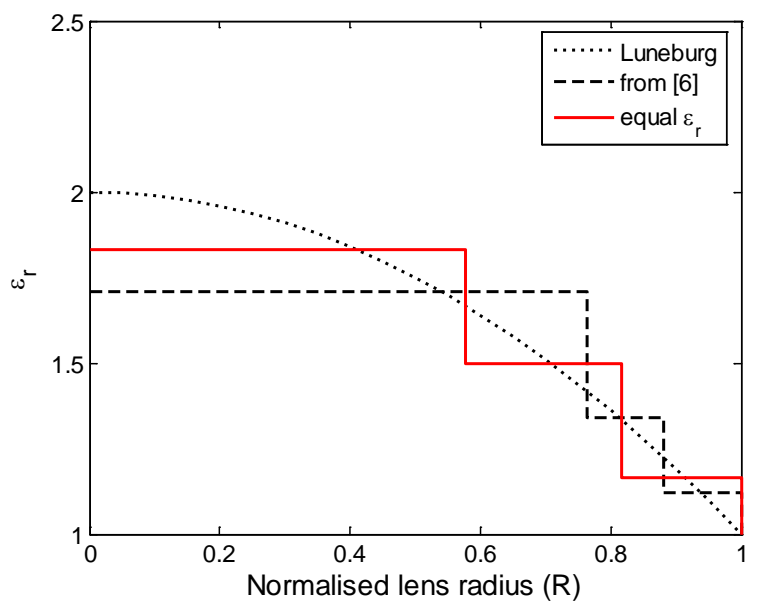

Fig. 5. Historical and equal $\varepsilon_{\mathrm{r}}$ step 3-layer simplfied Luneburg lens designs.

\section{SMALL RADII LUNEBURG LENSES}

Simplifying and thus reducing the manufacturing cost of Luneburg lenses by using a large homogeneous core was expounded in [6]. For the relatively small $2 \lambda_{0}$ radius class of lens considered here, a core of $\varepsilon_{\mathrm{r}}=1.71$ of radius $\mathrm{R}=0.764 \mathrm{R}_{0}$ was suggested, with 2 thin outer layers, Figure 5. Superficially, the design does not center on the Luneburg curve and appears to be biased toward higher $\varepsilon_{\mathrm{r}}$. For comparison, the Luneburg curve was discretized into 3 equal $\varepsilon_{\mathrm{r}}$ steps. Both 3-layer Luneburg lenses were simulated in FEKO $^{\mathrm{TM}}$ with a $140^{\circ} \mathrm{PEC}$ cap.

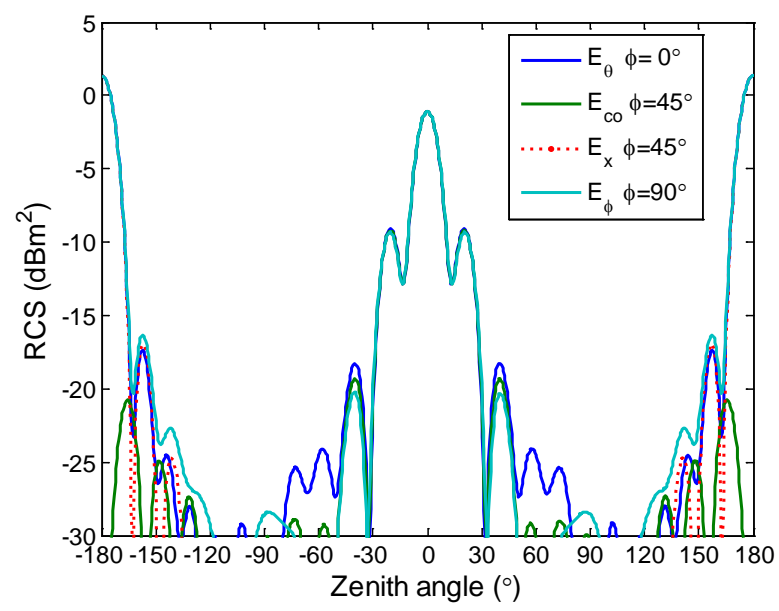

Fig. 6. Principal plane RCS patterns of 3-layer Luneburg reflector from [6]. 
In contrast to the total internal reflection lens, the 3-layer Luneburg lens produced a distinct and fully rotationally symmetric main beam consisting of a mainlobe and first sidelobes, Figure 6. Additionally, the $\phi=45^{\circ}$ plane crosspolarised component was more than $-30 \mathrm{~dB}$ below the mainlobe peak. The forward $\left(\theta=0^{\circ}\right)$ RCS was $-1.1 \mathrm{dBm}^{2}$. The equal $\varepsilon_{\mathrm{r}}$ step 3-layer Luneburg gave a forward RCS of $0.63 \mathrm{dBm}^{2}$, which is closer to the theoretical RCS value of $0.94 \mathrm{dBm}^{2}$ for a $2 \lambda_{0}$ radius spherical lens. The latter gave $1.7 \mathrm{~dB}$ better performance due to lower relative first sidelobe level.

\section{2-LAYER THORNTON LENS}

As described in the Introduction, the 2-layer xPS-PE lens was extensively evaluated as a medium gain antenna for communications systems. As a 2-layer spherical structure it is preferable to a 3 -layer structure. Here a $2 \lambda_{0}$ radius version was simulated in $\mathrm{FEKO}^{\mathrm{TM}}$, with the $\mathrm{xPS} \varepsilon_{\mathrm{r}}=2.53$ core extending from the sphere center to $\mathrm{R}=0.47 \mathrm{R}_{0}$, and the outer PE layer had $\varepsilon_{\mathrm{r}}=2.3$. The forward RCS was $1.5 \mathrm{dBm}^{2}$, exceeding the theoretical RCS value of $0.94 \mathrm{dBm}^{2}$. This good result is due to the first sidelobe level been $16.5 \mathrm{~dB}$ below the $\theta=0^{\circ}$ peak, which was close to $17 \mathrm{~dB}$ expected of a uniformly illuminated circular aperture.

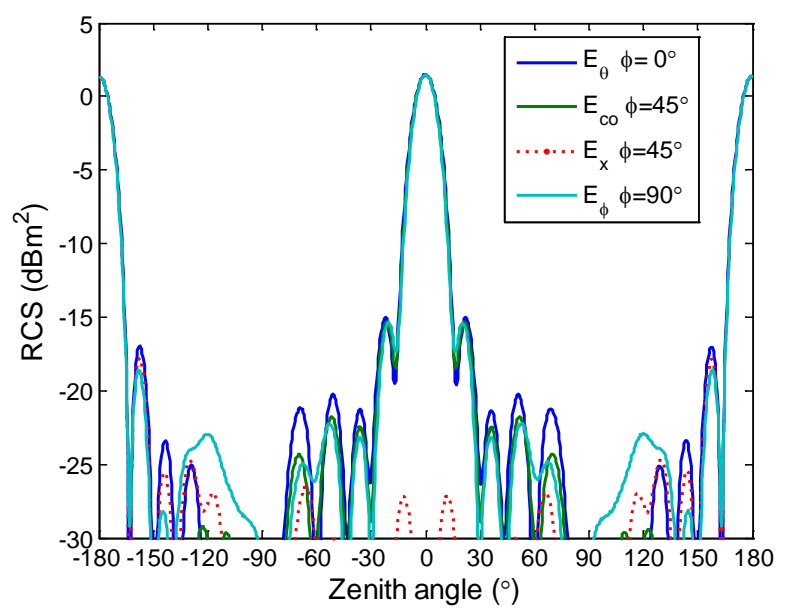

Fig. 7. Principal plane RCS patterns of 2-layer xPS-PE lens.

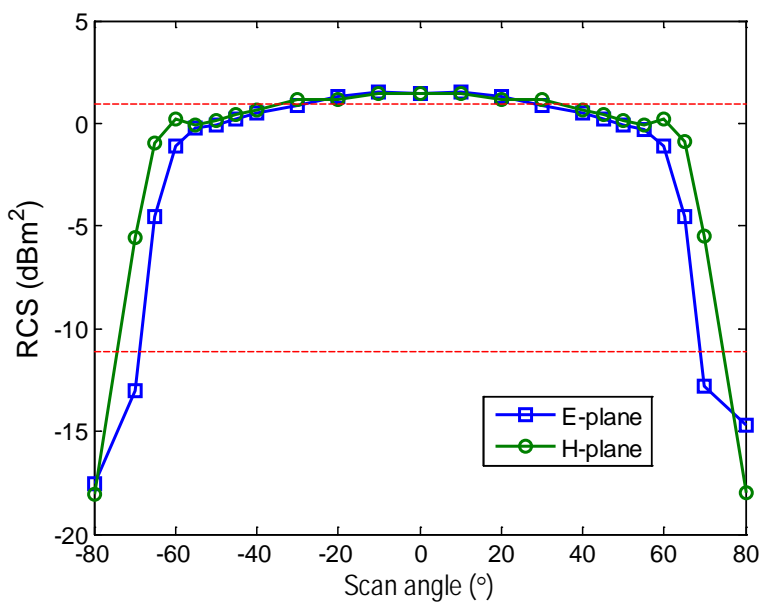

Fig. 8. Principal plane scan performance of 2-layer lens.
To check the practicality of the 2-layer xPS-PE lens, it was rotated $\pm 80^{\circ}$ in both the E-plane and the H-plane. As expected from prior work on Luneburg lenses, the scan range was limited by the $140^{\circ}$ metallic cap, Figure 8 . The E-plane $3 \mathrm{~dB}$ beamwidth was $123^{\circ}$ and the H-plane $3 \mathrm{~dB}$ beamwidth was $134^{\circ}$, which are satisfactory results.

If manufactured from xPS and PE, the $2 \lambda_{0}$ radius lens weight will be approximately 520 grams for $12 \mathrm{GHz}$. This weight is prohibitively high for a small platform like a quadrotor, but acceptable for man-portable use and larger platforms such as fishing boats.

\section{HOMOGENEOUS LENSES}

As a further simplification, a simple homogeneous sphere with $140^{\circ}$ metallic cap was simulated in $\mathrm{FEKO}^{\mathrm{TM}}$. The $\varepsilon_{\mathrm{r}}$ was varied from 1.7 to 3.5, Figure 9. Above $\varepsilon_{\mathrm{r}}=2.60$, the forward RCS was comparable to or exceeded the theoretical RCS value of $0.94 \mathrm{dBm}^{2}$. The peak RCS value was $2.7 \mathrm{dBm}^{2}$ for $\varepsilon_{\mathrm{r}}=2.90$. This result is peculiar to the relatively small $2 \lambda_{0}$ radius and is not expected to be repeated at larger radii where the phase error in a homogeneous lens will become significant [6].

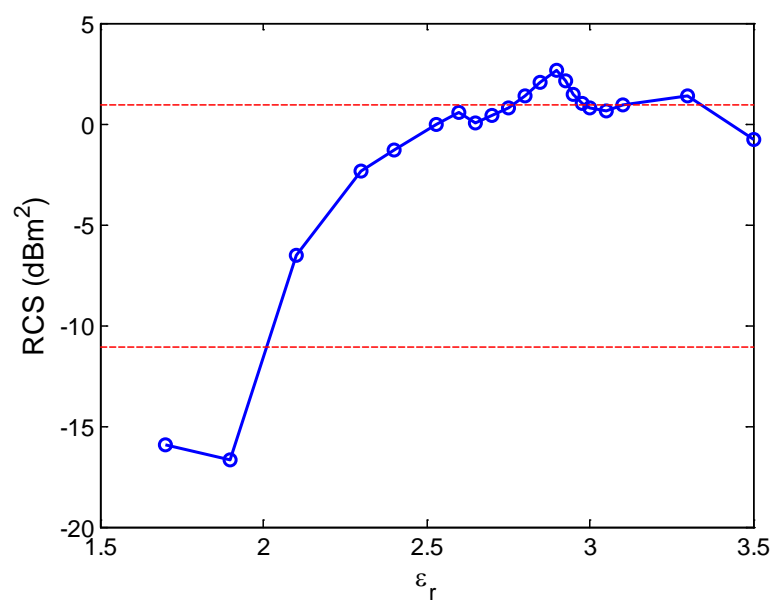

Fig. 9. Effect of $\varepsilon_{\mathrm{r}}$ on RCS of $2 \lambda_{0}$ radius homogeneous sphere.

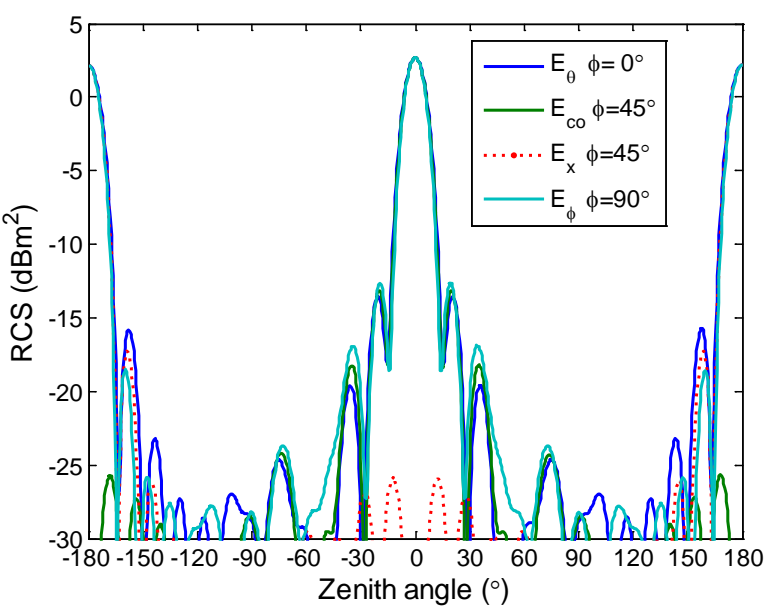

Fig. 10. Principal plane RCS patterns of $\varepsilon_{\mathrm{r}}=2.90$ homogeneous lens.

Similar to the 2-layer xPS-PE lens, a good forward RCS value was given by the $\varepsilon_{\mathrm{r}}=2.90$ lens as it produced a good rotationally symmetric main beam having first sidelobe level 
15.3dB below the $\theta=0^{\circ}$ peak, and the $\phi=45^{\circ}$ plane crosspolarised component was about $-30 \mathrm{~dB}$ below peak.

The scan performance of the 3-layer Luneburg lenses and the $\varepsilon_{\mathrm{r}}=2.90$ homogeneous lens will be given in the full paper.

\section{ACKNOWLEDGMENT}

This work was started at NICT Satellite Communications Group under the direction of Dr. R. Suzuki during the 2009/10 fiscal year.

\section{REFERENCES}

[1] D. Bird, "Design and manufacture of a low-profile radar retroreflector," presented at the RTO SCI Symposium on Sensors and
Sensor Denial by Camouflage, Concealment and Deception, Brussels, Belgium, 19-20 April 2004, RTO-MP-SCI-145.

[2] F.T. Ulaby, "Measurement of soil moisture content," CRES Technical Report 177-35, April 1973.

[3] D. Gray, J. Thornton, H. Tsuji \& Y. Fujino, "Scalar feeds for 8 wavelength diameter homogeneous lenses," IEEE Int. Ant. and Prop. Symp., North Charleston, June 2009.

[4] J. Thornton, "Wide-scanning multi-layer hemisphere lens antenna for Ka band", IEE Proc.-Microw. Antennas Propag., Vol. 153, No. 6, December 2006.

[5] N. Ochai, "Omnidirectional dielectric lens reflector," United States patent 3,550,147, granted Dec. 22, 1970.

[6] N. Ochiai, "Shell-type luneberg lens," United States patent 3,465,362, granted Sep. 2, 1969. 